\title{
A Generalization of Discounted Central Limit Theorem and its Berry-Esseen Analogue
}

\author{
B. Amiri Aghbilagh ${ }^{1}$, Z. Shishebor ${ }^{1}$, M.M. Saber ${ }^{1}$ \\ ${ }^{1}$ Department of Statistics, Faculty of Science, Shiraz University, Shiraz 71454, Iran \\ behnam.amiri.a@gmail.com, sheshebor@susc.ac.ir,mohammadmehdisaber@gmail.com \\ Received 12 March 2014 \\ Accepted 11 July 2014
}

\begin{abstract}
Discounted central limit theorem was proved for i.i.d. random variables by Gerber (Theorem 2.1). This work is intended to extend the work of Gerber to the case where $\left\{X_{i}\right\}$ is a periodic sequence of independent variables and $v_{\mathrm{i}}$ are periodic scalars. A simulation study was performed in order to support the result. We also give an interpretation of our work in a financial context. Finally, an example with real data is presented.
\end{abstract}

Keywords: Berry-Esseen Theorem; Discount factor; Present value.

\section{Introduction}

In mathematical statistics, approximation theorems that provide an upper bound for the error of approximation are always of great importance. Among these theorems, Berry-Essen Theorem, which is stated by Berry (1941) and Esseen (1945), gives an upper bound to the error of central limit theorem approximation for the class of distributions for which $\rho=E|X-\mu|^{3}<\infty$, [1] and [4]. In 1971 Gerber proved that if $X_{v}=\sum_{k=0}^{\infty} v^{k} X_{k}$, where $X_{1}, X_{2}, \ldots$ is a sequence of independent random variables, $0<v<1$, and the first three moments of $X_{k}$ are finite, then there exists an upper bound for the error of approximation of distribution of $X_{v}$ by the normal distribution, [6]. Besides, he pointed out that his theorem is analogous to the Berry-Esseen Theorem and called it the discounted version of the Berry-Esseen Theorem. As its application he showed that the normalized random variable

$$
Z_{v}=\frac{(1-v)^{\frac{1}{2}}}{\sigma}\left(X_{v}-\frac{\mu}{1-v}\right)
$$

is asymptotically normal as $v \rightarrow 1$. He mentioned that $X_{v}$ may be interpreted as the present value of the sum of certain periodic and identically distributed payments, $X_{k}$, with discount factor $v$. Recent research in this field is done by Saulis and Deltuviene (2006), in which a non-uniform estimate of the Berry-Esseen Theorem in a discounted version is proved, [9].

In economic literature the present value is an important financial concept; it describes the process of determining what a cash flow to be received in the future is worth in today's money. In many practical economic problems the payments are random variables and consequently the present value is also a random variable. Thus, knowing about the distribution of present value is 
essential for statistical inference. There are many problems in mathematical finance which require the evaluation of present value in the case that the discount factors and the values of payments are periodic. This was our motivation for current work. In this work we attempt to extend the result of Gerber to the case where $\left\{X_{i}\right\}$ is a sequence of independent random variables and $X_{i+T k}$ has the same distribution as $X_{i}$ for a fixed constant, $T \in \mathbb{N}, 0<v_{i}<1, i=0, \ldots, T-1$.

\section{Main Result}

To begin this section we first state the Gerber Theorem. In the sequel, let $F_{X_{\gamma}}$ and $\Phi_{\mu, \sigma^{2}}$ denote the distribution functions of $X_{\gamma}$ and the Normal distribution with mean $\mu$ and variance $\sigma^{2}$, respectively.

Theorem 2.1. (H. Gerber) Let $\left\{X_{k}\right\}_{k=0}^{\infty}$ be a sequence of independent random variables with common distribution function $F$. Let $v$ be a discount factor $(0<v<1)$ and assume that the first three moments of $X_{k}$ are finite:

$$
\mu=\int_{-\infty}^{+\infty} x d F(x)<\infty, \quad \sigma^{2}=\int_{-\infty}^{+\infty}(x-\mu)^{2} d F(x)<\infty, \quad \rho=\int_{-\infty}^{+\infty}|x-\mu|^{3} d F(x)<\infty .
$$

If $X_{v}=\sum_{k=0}^{\infty} v^{k} X_{k}$ and $Z_{v}=\frac{(1-v)^{\frac{1}{2}}}{\sigma}\left(X_{v}-\frac{\mu}{1-v}\right)$, then for all $x$,

$$
\left|F_{Z_{v}}(x)-\Phi_{0,(1+v)^{-1}}(x)\right| \leq C(1-v)^{\frac{1}{2}}\left(\rho / \sigma^{3}\right)
$$

where

$$
C=\left[\frac{1}{\sqrt{\pi}}\left(\frac{5}{24} \frac{1}{1+v+v^{2}}\right)\left(\frac{1}{2} \frac{1}{1+v}-\frac{5}{12} \frac{1}{1+v+v^{2}}\right)^{-\frac{3}{2}}+\frac{24}{\pi} \frac{(1+v)^{\frac{1}{2}}}{\sqrt{2 \pi}}\right] .
$$

If $0.9 \leq v \leq 1$, then the value 5.4 is an upper bound for $C$.

Before proceeding to the proof of Theorem 2.2, which is the main result of this paper, we need the following lemma.

Lemma 2.1. If $F_{i}, G_{i}, i=1, \ldots, n$, are distribution functions and $a_{i}, i=1, \ldots, n$, are fixed numbers and if

$$
\left|F_{i}(x)-G_{i}(x)\right| \leq a_{i} \quad \forall x \in \mathbb{R}, i=1, \ldots, n,
$$

then

$$
\left|F_{1} * F_{2} * \ldots * F_{n}(x)-G_{1} * G_{2} * \ldots * G_{n}(x)\right| \leq \sum_{i=1}^{n} a_{i},
$$

where $*$ denotes the convolution operation of functions.

Proof. The proof is by induction on $n$. Since 


$$
\begin{aligned}
\left|F_{1} * F_{2}(x)-G_{1} * F_{2}(x)\right| & =\left|\int_{-\infty}^{+\infty} F_{1}(x-t) d F_{2}(t)-\int_{-\infty}^{+\infty} G_{1}(x-t) d F_{2}(t)\right| \\
& \leq \int_{-\infty}^{+\infty}\left|F_{1}(x-t)-G_{1}(x-t)\right| d F_{2}(t) \leq a_{1}
\end{aligned}
$$

thus

$$
\left|F_{1} * F_{2}(x)-G_{1} * F_{2}(x)\right| \leq a_{1} .
$$

We have

$$
\begin{aligned}
\left|F_{1} * F_{2}(x)-G_{1} * G_{2}(x)\right|= & \frac{1}{2}\left|2 F_{1} * F_{2}(x) \pm G_{2} * F_{1}(x) \pm G_{1} * F_{2}(x)-2 G_{1} * G_{2}(x)\right| \\
\leq & \frac{1}{2}\left[\left|F_{1} * F_{2}(x)-G_{1} * F_{2}(x)\right|+\left|F_{1} * F_{2}(x)-G_{2} * F_{1}(x)\right|\right. \\
& \left.+\left|G_{2} * F_{1}(x)-G_{1} * G_{2}(x)\right|+\left|G_{1} * F_{2}(x)-G_{1} * G_{2}(x)\right|\right] \\
\leq & a_{1}+a_{2} .
\end{aligned}
$$

This gives the proof for the case $n=2$. Assuming (2.1) to hold for $k$, by a similar argument one can show that it is true for $k+1$.

Theorem 2.2. Let $\left\{X_{k}\right\}_{k=0}^{\infty}$ be a sequence of independent random variables. Moreover assume that $X_{i+T k}$ has the same distribution as $X_{i}$ and $v_{i+T k}=v_{i}$ for a fixed constant, $T \in \mathbb{N}, i=$ $0, \ldots, T-1$. Suppose that the first three moments of $X_{i}, i=0, \ldots, T-1$ are finite i.e.

$\mu_{i}=\int_{-\infty}^{+\infty} x d F_{X_{i}}(x)<\infty, \sigma_{i}{ }^{2}=\int_{-\infty}^{+\infty}\left(x-\mu_{i}\right)^{2} d F_{X_{i}}(x)<\infty, \rho_{i}=\int_{-\infty}^{+\infty}\left|x-\mu_{i}\right|^{3} d F_{X_{i}}(x)<\infty$,

and $\quad \sum_{k=0}^{\infty} v_{k}{ }^{k}\left|X_{k}\right|<\infty . \quad$ Let $\quad \boldsymbol{v}=\left(v_{0}, v_{1}, \ldots, v_{T-1}\right), \quad X_{\boldsymbol{v}}=\sum_{k=0}^{\infty} v_{k}{ }^{k} X_{k} \quad$ and $Z_{\boldsymbol{v}}=\sqrt{1 / \sum_{i=0}^{T-1} \frac{\sigma_{i}{ }^{2} v_{i}^{2 i}}{1-v_{i}{ }^{2 T}}}\left(X_{\boldsymbol{v}}-\sum_{i=0}^{T-1} \frac{v_{i}{ }^{i} \mu_{i}}{1-v_{i}{ }^{T}}\right)$. Then

$$
\left|F_{Z_{v}}(x)-\Phi_{0,1}(x)\right| \leq \sum_{i=0}^{T-1} C_{i}\left(1-v_{i}^{T}\right)^{\frac{1}{2}}\left(\rho_{i} / \sigma_{i}{ }^{3}\right),
$$

where

$$
C_{i}=\left[\frac{1}{\sqrt{\pi}}\left(\frac{5}{24} \frac{\left(1+v_{i}^{T}\right)^{\frac{3}{2}}}{1+v_{i}^{T}+v_{i}^{2 T}}\right)\left(\frac{1}{2}-\frac{5}{12} \frac{1+v_{i}^{T}}{1+v_{i}^{T}+v_{i}^{2 T}}\right)^{-\frac{3}{2}}+\frac{24}{\pi} \frac{\left(1+v_{i}^{T}\right)^{\frac{1}{2}}}{\sqrt{2 \pi}}\right] .
$$

Proof. Let $X_{v_{i}}=\sum_{k=0}^{\infty} v_{i}^{T k} X_{i+T k}$ and

$$
Z_{v_{i}}=\frac{\left(1-v_{i}^{2 T}\right)^{\frac{1}{2}}}{\sigma_{i}}\left(X_{v_{i}}-\frac{\mu_{i}}{1-v_{i}^{T}}\right) .
$$

Then we get immediately the following relation from Theorem 2.1 for, $i=0, \ldots, T-1$. 


$$
\left|F_{Z_{v_{i}}}(x)-\Phi_{0,1}(x)\right| \leq C_{i}\left(1-v_{i}^{T}\right)^{\frac{1}{2}}\left(\rho_{i} / \sigma_{i}^{3}\right) \quad \forall x \in \mathbb{R}
$$

From (2.2) we have $Z_{v}=\sum_{i=0}^{T-1} t_{i} Z_{v_{i}}$ with $t_{i}=\sigma_{i} v_{i}{ }^{i} /\left(\left(1-v_{i}{ }^{2 T}\right)^{\frac{1}{2}} \sqrt{\sum_{j=0}^{T-1} \frac{v_{j}^{2 j} \sigma_{j}^{2}}{1-v_{j}^{2 T}}}\right)$. It is clear that $F_{t_{i} Z_{v_{i}}}()=.F_{Z_{v_{i}}}\left(\dot{\overline{t_{i}}}\right)$. The relation (2.3) leads us to

$$
\left|F_{t_{i} Z_{v_{i}}}(x)-\Phi_{0, t_{i}{ }^{2}}(x)\right|=\left|F_{Z_{v_{i}}}\left(\frac{x}{t_{i}}\right)-\Phi_{0,1}\left(\frac{x}{t_{i}}\right)\right| \leq C_{i}\left(1-v_{i}^{T}\right)^{\frac{1}{2}}\left(\frac{\rho_{i}}{\sigma_{i}^{3}}\right) .
$$

Since $\sum_{i=0}^{T-1} t_{i}^{2}=1, \Phi_{0,1}(x)=\Phi_{0, t_{0}^{2}} * \Phi_{0, t_{1}^{2}} * \ldots * \Phi_{0, t_{T-1}^{2}}(x)$. From independence of $X_{i}$ we have $F_{Z_{v}}(x)=F_{t_{0} Z_{v_{i}}} * \ldots * F_{t_{T-1} Z_{v_{i}}}(x)$. From what we have just stated, Lemma 2.1, and (2.4), we have

$$
\left|F_{Z_{v}}(x)-\Phi_{0,1}(x)\right| \leq \sum_{i=0}^{T-1} C_{i}\left(1-v_{i}{ }^{T}\right)^{\frac{1}{2}}\left(\rho_{i} / \sigma_{i}{ }^{3}\right) .
$$

This completes the proof.

Remark: An immediate consequence of this theorem gives us that the normalized random variable $Z_{\boldsymbol{v}}$ is asymptotically normal as $\boldsymbol{v}=\left(v_{0}, v_{1}, \ldots, v_{T-1}\right) \rightarrow(1,1, \ldots, 1)$.

The random variable $X_{\boldsymbol{v}}$ can be interpreted as the present value in the case that $v_{i}$ and $X_{i+T k}$ are discount factor and the value of payment, respectively, which are related to the season.

\section{Simulation Study}

To validate the result of the previous section we present a simulation study. We have performed a simulation study for different statistical distributions, different $\boldsymbol{v}$ 's and sample sizes, for $T=$ $4,6,12$. Here is an overview of our approach.

Step 1: $T$ different statistical distributions were chosen, the arbitrary values $v_{0}, \ldots, v_{T-1}$ were considered. We let $v_{i}$ 's tend to 1 .

Step 2: A random sample of size $n,\left\{x_{i}, x_{i+T}, \ldots, x_{i+(n-1) T}\right\}$, was drawn from the $i$ th distribution.

Step 3: The following was computed: $X_{\boldsymbol{v}}=\sum_{k=0}^{n T-1} v_{k}{ }^{k} X_{k}$.

Step 4: The second and third steps were repeated 1000 times, then the empirical distribution function of $Z_{v}$ was obtained and was named $F_{n}$.

Step 5: The normality test was employed using Kolmogorov-Smirnov statistic $D_{n}=$ $\sup _{x}\left|F_{n}(x)-\Phi_{0,1}(x)\right|$ at level of significance 0.01 . 
Our observations demonstrated that by increasing $\boldsymbol{v} \rightarrow \mathbf{1}$ the accuracy of approximation in most cases increased; indeed the p-values were greater than 0.01 . Therefore, the hypothesis of normality is not rejected as $\boldsymbol{v} \rightarrow \mathbf{1}$. Figure 1 displays the graphs of the distribution functions of $Z_{v}$ for $T=4, X_{1} \sim \operatorname{Pos}(1), X_{2} \sim G e(0.2), X_{3} \sim \operatorname{Bin}(15,0.75), X_{4} \sim H G(10,10,5)$, with different $\boldsymbol{v}$ 's. It can be seen that, as $\boldsymbol{v} \rightarrow \mathbf{1}$, the distribution function of $Z_{\boldsymbol{v}}$ converges to the distribution function of standard normal. In a similar way, two plots are shown for $T=6$ and $T=12$ in Figure 2 and Figure 3, respectively.

\section{Empirical Evidence}

We will here draw out an implication for a relevant important topic, investment appraisal. As we know one of the most important responsibilities of the plan management in financial economics is asset allocation decision, [7]. This section contains an investment appraisal for a white meat manufacturing and packing firm. Figure 4 shows quarterly expected profit that is collected from a study to setup a new factory in Iran. The initial cost of investment for this firm is 155.8 Billion Iranian Rials. We used expected quarterly data from the first quarter of 2014 until fourth quarter of 2023.

In well-developed capital markets, interest rates will reflect expectations of inflation, and hence it will not make much difference whether we measure the alternative cost of capital by interest rates or inflation rates, [2]. But when capital markets are not free because interest rates are subject to regulations or have ceilings -such as Iran- it is often appropriate to use inflation, not interest rates, as the measure of the alternative cost, [3]. It is believed that the right measure of the opportunity cost of capital is the higher of the two: interest rates or inflation, [8]. Hence, to calculate present value, we discount expected profit by the inflation rate.

For $n$th $^{1}$, year we employed a test (based on the statistic $X_{v}$ with significance level of 0.01) to test the null hypothesis, $H_{0}: \mu_{v} \leq A$, against $H_{1}: \mu_{v}>A$. The alternative hypothesis shows the year in which the average present value, $\mu_{v}$, is greater than the initial investment, $A$, of the firm. In fact, this test statistically represents the year in which the initial cost of investment returns to the investors. Rejection of the hypothesis $H_{0}$ indicated that in year $n$ the average present value is greater than the initial investment. Hence, for small $n$ the decision maker should invest in this project.

Table 1 reports the results. This table shows the year of capital return based on the initial cost of investment. As we already mentioned the initial costs of investment of the firm is 155.8 billion Iranian Rials, which is between 105 and 175 . Hence, the year of capital return is the third year. Our test shows that it is profitable that the decision maker allocates 155.8 billion Iranian Rials to this project.

\footnotetext{
${ }^{1}$. This year should be before the useful lifetime of the investment project.
} 


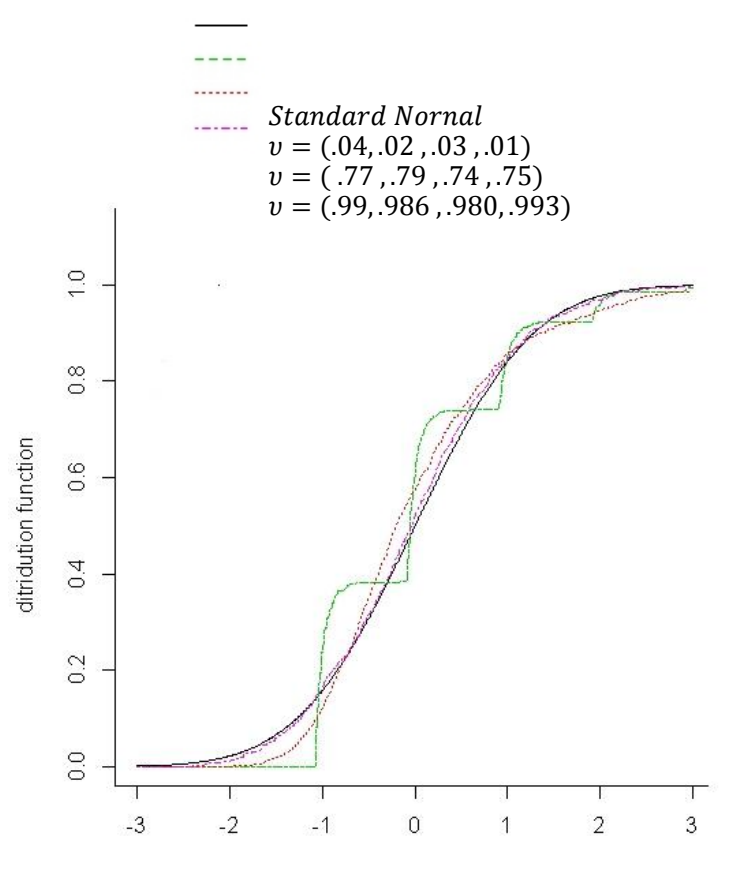

Figure 1. $T=4$ and $X_{1} \sim \operatorname{pos}(1), X_{2} \sim G e(0.2)$, $X_{3} \sim \operatorname{Bin}(15,0.75), X_{4} \sim H G(10,10,5)$

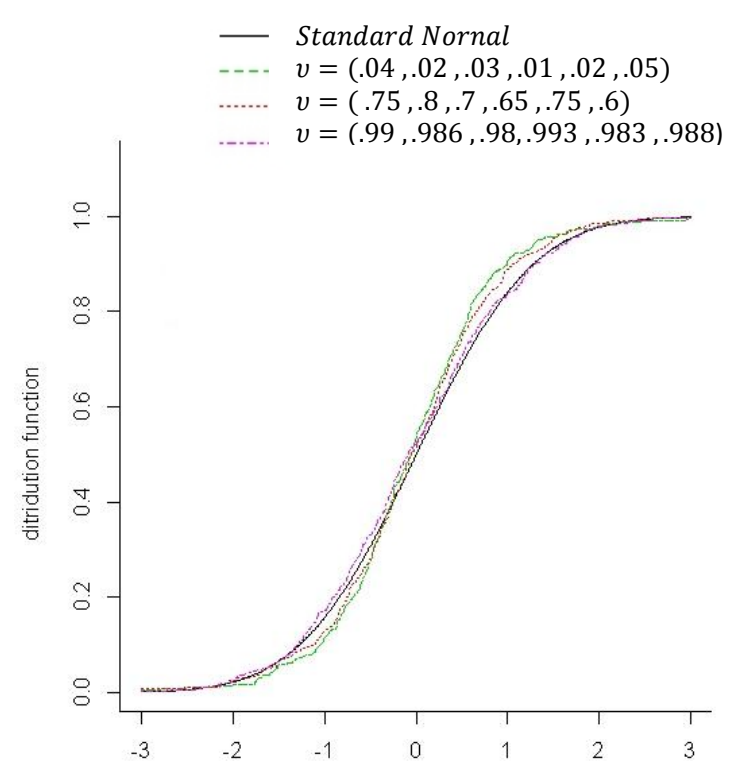

Figure 2. $T=6$ and $X_{1} \sim t(4), X_{2} \sim t(8), X_{3} \sim t(10)$, $X_{4} \sim t(12), X_{5} \sim t(15), X_{6} \sim t(6)$,

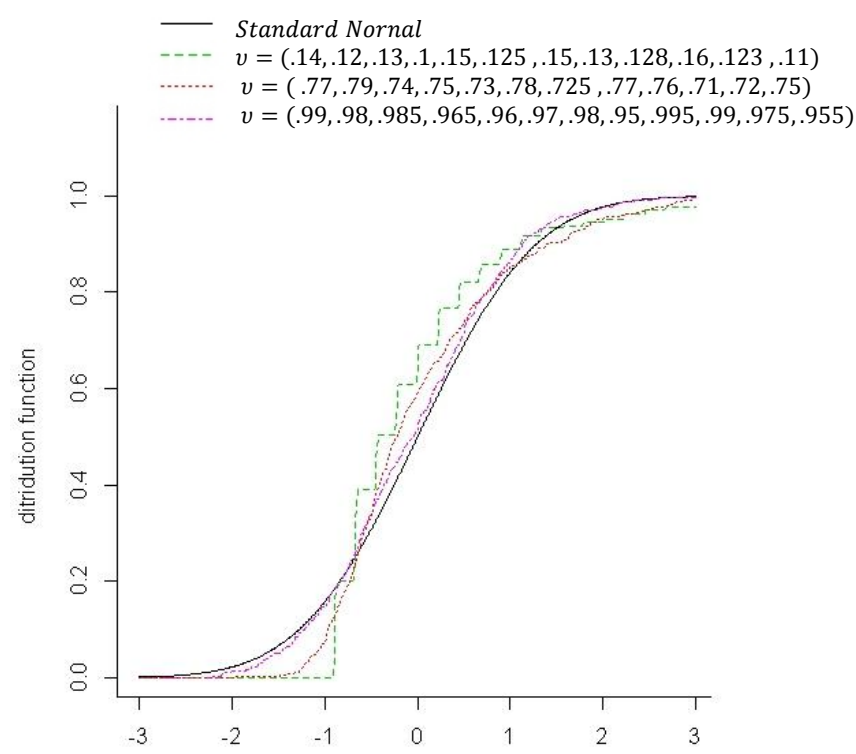

Figure 3. $\mathrm{T}=12$ and $\mathrm{X}_{1} \sim \mathrm{Ge}(0.2), \mathrm{X}_{2} \sim \mathrm{E}(5), \mathrm{X}_{3} \sim \operatorname{Bin}\left(20, \frac{1}{4}\right)$, $\mathrm{X}_{4} \sim \mathrm{t}(4), \mathrm{X}_{5} \sim \operatorname{Pos}(5), \mathrm{X}_{6} \sim \mathrm{U}(5,10), \mathrm{X}_{7} \sim \mathrm{E}(1), \mathrm{X}_{8} \sim \operatorname{Pos}(4)$, $X_{9} \sim t(9), X_{10} \sim t(11), X_{11} \sim \operatorname{Bin}\left(15, \frac{3}{4}\right), X_{12} \sim U(3,6)$.

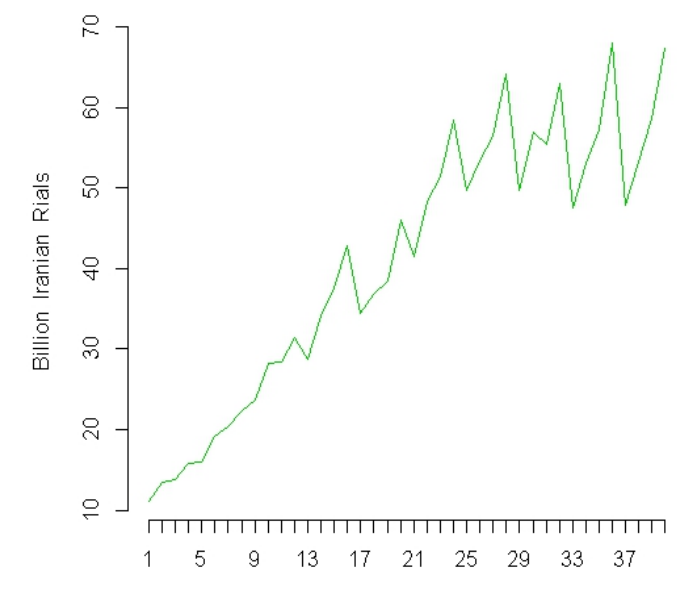

Figure 4. Quarterly expected profit for a white meat manufacturing and packing firm January 2104 December 2023 
Table 1. Results of the test

\begin{tabular}{|c|c|c|c|c|c|c|c|}
\hline $\begin{array}{c}\text { Range of Initial } \\
\text { Costs of Investment } \\
\text { (Billion Iranian } \\
\text { Rials) }\end{array}$ & $45-100$ & $105-170$ & $175-250$ & $255-320$ & $325-395$ & $400-470$ & $475-530$ \\
\hline $\begin{array}{c}\text { Year of Capital } \\
\text { Return }\end{array}$ & 2 & 3 & 4 & 5 & 6 & 7 & 8 \\
\hline
\end{tabular}

\section{Acknowledgements}

The authors would like to thank the two anonymous referees for carefully reading the paper and for their comments which greatly improved the paper. The authors are also grateful to Porf. Reza Moosavi Mohseni for providing the real example used in this paper.

\section{References}

[1] A. C. Berry, The accuracy of the Gaussian approximation to the sum of independent variates, Trans. Amer. Math. Soc. 49 (1941) 122-136.

[2] P. Cagan, The monetary dynamics of hyperinflation. in Milton Friedman [ed.]. Studies in the quantity theory of money, (Chicago: University of Chicago Press 1956).

[3] R. Dornbusch and S. Fischer, Macroeconomics. 5th edition, (New York: McGraw-Hill publishing company 1990).

[4] C. G. Esseen, Fourier analysis of distribution functions. A mathematical study of the Laplace Gaussian law, Acta Math. 77 (1945) 1-125.

[5] W. Feller, An introduction to probability theory and its applications. Vol. II, (John Wiley \& Sons, Inc, New York-London-Sydney 1966).

[6] H. U. Gerber, The discounted central limit theorem and its Berry-Esseen analogue, Annals of Mathematical Statistics. 42 (1971) 389-392.

[7] L. J. Halliwell, Conjoint prediction of paid and incurred losses, Insurance: Mathematics and Economics. 22(2) (1997) 175-176.

[8] L. Papademos and F. Modogliani, Inflation, financial and fiscal structure, and the monetary mechanism, European Economic Review. 21 (1983) 203-250.

[9] L. Saulis and D. Deltuviene, The discounted limit theorems, Acta Appl. Math. 3 (2006) 219-226. 